\section{Early-onset dementia and extrapyramidal disease: clinicopathological variant of Gerstmann-Straussler-Scheinker or Alzheimer's disease?}

\author{
John Hart Jr, Barry Gordon
}

tical atrophy with moderate ventricular dilatation, and symmetric basal ganglia calcifications on computerised tomography (CT). EEG and CSF were normal except for a CSF protein of $66 \mathrm{mg} / \mathrm{dl}$.

By the age of 38 , his dementia was worse, including impaired language. There was oral/ facial apraxia, rigidity of all extremities, dystonic posturing of the arms and hands with choreiform movements of the fingers, mild intention tremor, and flexed, short-stepped gait (without evidence of gait apraxia). There was hyporeflexia in the lower extremities, with extensor plantar reflexes. Extensive laboratory evaluation was remarkable only for a CSF protein of $50 \mathrm{mg} / \mathrm{dl}$ (normal 15-45), CSF IgG index of 1.2 (normal $0 \cdot 2-0.8$ ), and EEG with poorly organised 7-8/s alpha and intermittent left fronto-centro-temporal theta and delta activity.

He continued to deteriorate, and died at the age of 39 years from bilateral aspiration pneumonia.

Interest has recently been rekindled in the interrelationships between Creutzfeldt-Jakob disease (CJD), Gerstmann-Straussler-Scheinker disease (GSS), and Alzheimer's disease (AD). Kuru-like cerebellar plaques are typical in CJD as well as GSS, and also occur in rare cases of $\mathrm{AD}^{1}{ }^{1}$ spongiform encephalopathy typical of CJD can be transmitted to monkeys from some cases of GSS. ${ }^{2}$ On this and other evidence $^{3}$ it has been suggested that $A D, G S S$ and CJD share a common pathogenic expression, or perhaps even a common aetiology. ${ }^{2}$ Cases that have various features of both GSS and $\mathrm{AD},{ }^{4}$ such as the one reported here, provide additional data about these interrelationships.

\section{Case report}

A 29 year old right handed white male gradually developed emotional lability, stiffening of his arms and legs, facial grimacing, memory loss, and difficulty learning new skills, over a two year period. Memory and general intellectual abilities gradually declined over the next four to five years. Family history was negative except for two brothers with mild essential tremor by the ages of 43 and 51 years.

At the age of 35, neurological examination showed moderate dementia (Verbal IQ $=81$, Performance IQ $=62$ ) with impaired memory and poor insight, dystonic posturing of both hands (left more than right), and slightly flexed gait. There was diffuse, moderate cor-

\section{Neuropathology}

Gross examination. Only the brain was available. It weighed 1200 grams. Vessels appeared normal. There was mild cortical atrophy, mildly dilated lateral ventricles, and hypopigmented substantia nigra and locus ceruleus.

Microscopic examination. Representative tissue blocks from the CNS were stained with haematoxylin-eosin, haematoxylin-eosin/ LFB, PTAH, Congo red, and NaumenkoFeigin/PAS stains to define plaques, amyloid deposits, neurofibrillary tangles, nuclei, and gliosis. The overwhelmingly predominant neuropathological finding was numerous

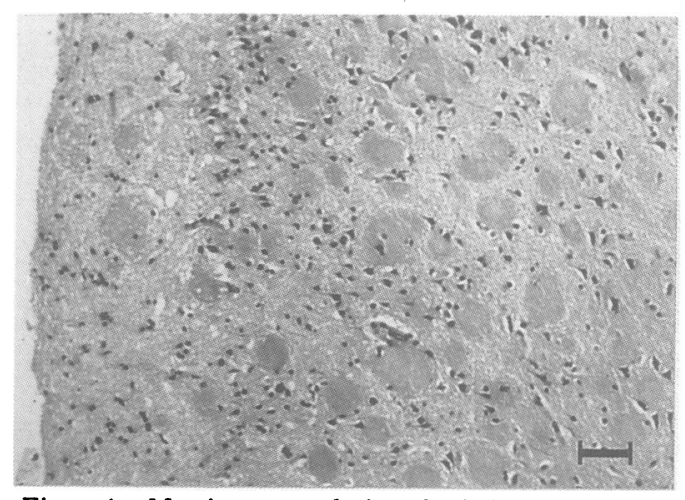

Figure 1 Massive accumulation of primitive plaques resulting in disruption of the cytoarchitecture and neuronal loss is evident in this section from the temporal lobe $(H \mathcal{E} E)$. The scale bar represents 100 microns. 


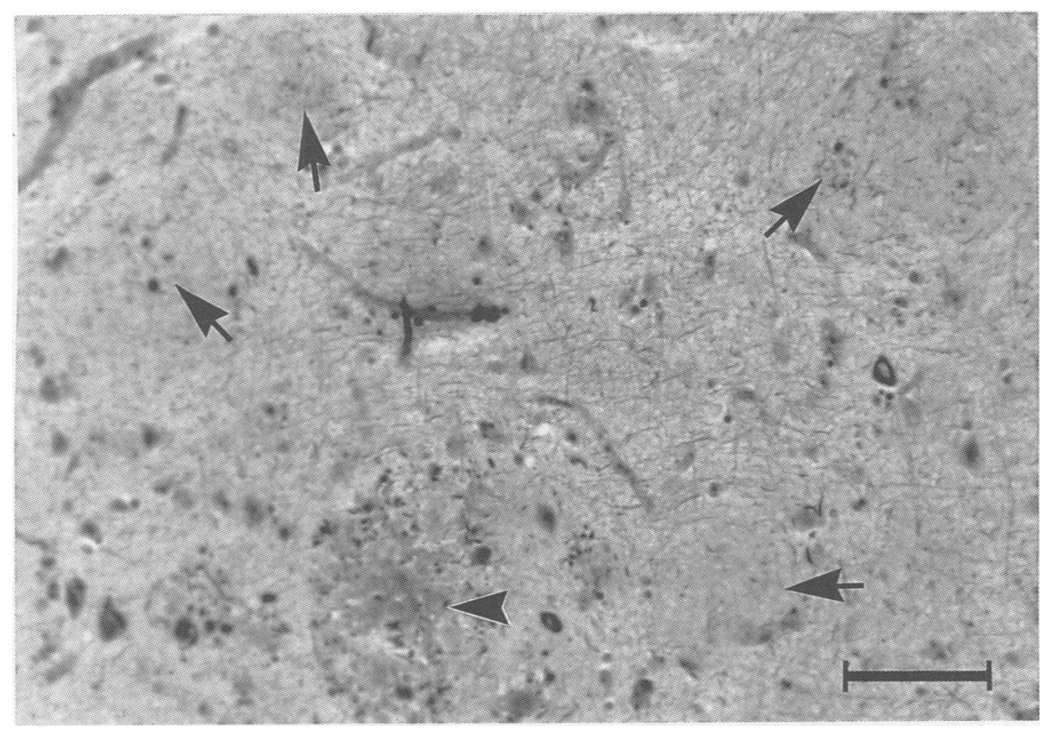

Figure 2 On silver stain, the primitive plaques (arrows) show a variable neuritic component but are devoid of an amyloid core. In contrast, a mature senile plaque (arrowhead) has a central core of amyloid (Naumenko-Feigin/PAS). The scale bar represents $75 \mu$.

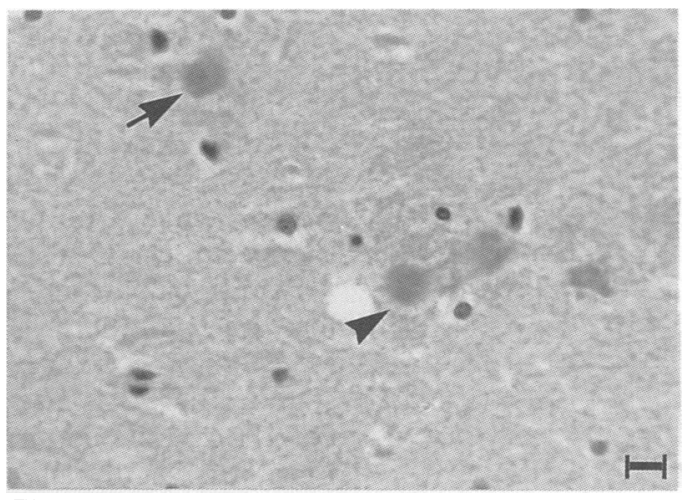

Figure 3 Kuru (arrow) and multicentric (arrowhead) plaques are seen in the molecular layer of the cerebellum $(H \in E)$. The scale bar represents 10 microns.

pus, striatum, and the superficial cortical layers of the neocortex. There were scant plaques in the thalamus and in the midbrain away from the substantia nigra, and a few plaques were located in the midbrain tegmentum. Senile plaques (fig 2) were most prominent in the amygdala and hippocampus and less abundant in the neocortex and dien-

polymorphic plaques throughout the cerebrum and subcortical structures which consisted of four types: primitive, senile, kuru, and multicentric plaques. Primitive plaques (fig 1, 2) were located in the cerebrum, diencephalon, and brainstemmost prominent in the amygdala, hippocam- cephalon. Kuru-type and multicentric amyloid plaques (fig 3) were seen in the cerebellum in the molecular, purkinje, and superficial granular cell layers. These plaques were also present in the deeper layers of the cortex and subcortical white matter, particularly in the occipital regions.

Table Summary of clinical and neuropathological features of patients with characteristics of GSS and AD

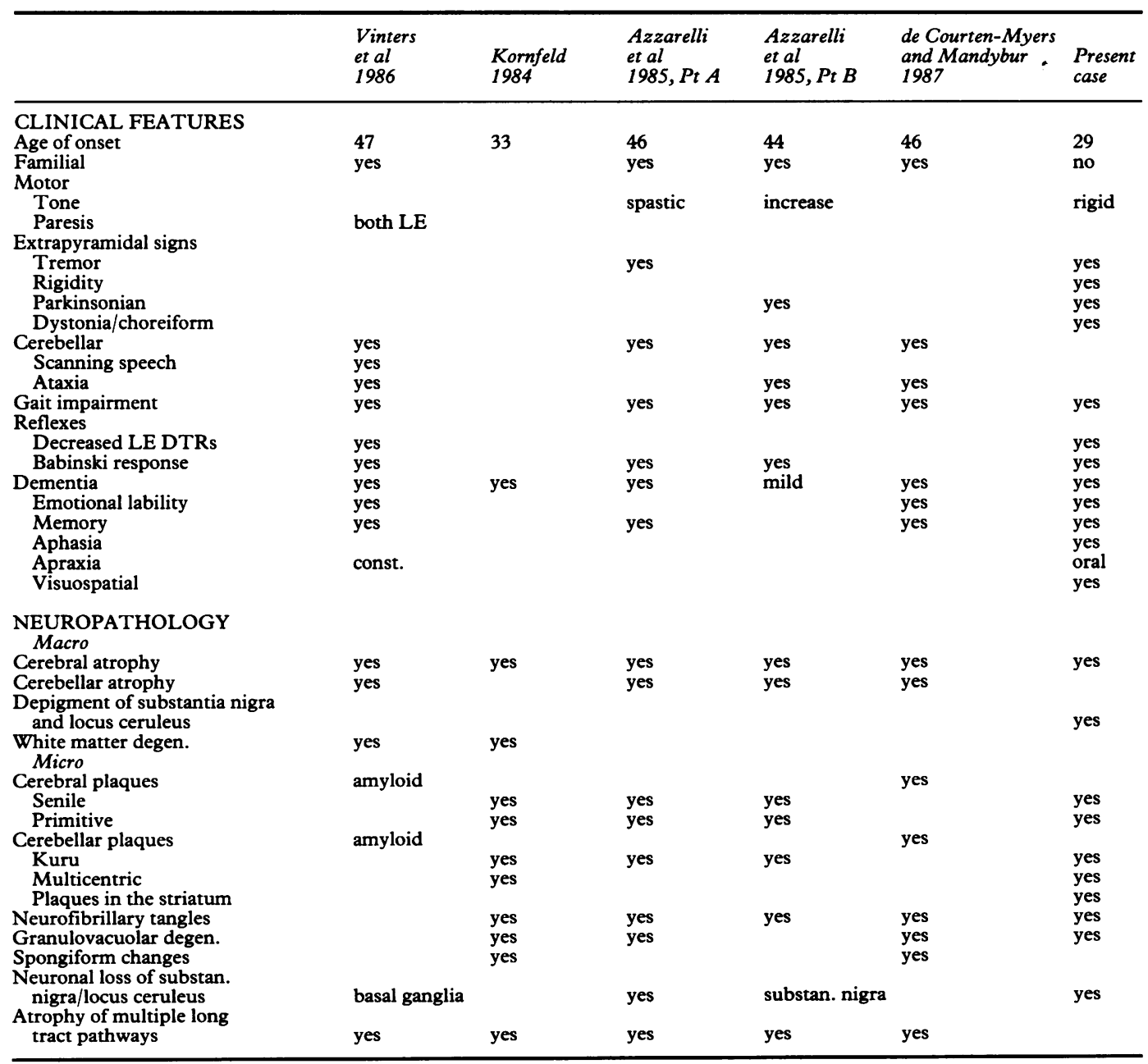


Moderate numbers of neurofibrillary tangles were seen throughout the neocortex, amygdala, and hippocampus. They were not noted in other subcortical regions or the substantia nigra. Granulovacuolar degeneration was present in the pyramidal neurons of the hippocampus.

Additionally, there was subjective neuronal loss, especially in regions containing plaques, as determined from $\mathrm{H} \& \mathrm{E}$ and $\mathrm{H} \& \mathrm{E} / \mathrm{LFB}$ stains. There was also mild gliosis as assessed with $\mathrm{H} \& \mathrm{E}$ and confirmed with PTAH stains. Both the neuronal loss and gliosis were present in the cerebrum, diencephalon, and brainstem, most prominently in the amygdala, hippocampus, and neocortex. In some of these areas, there was mild rarefaction of the neuropil but no spongiform change. There was also mild neuronal loss in the substantia nigra and locus ceruleus with extracellular pigment deposition. No Lewy bodies were noted in the substantia nigra.

Mild congophilic angiopathy was seen in the superficial vessels of the cortex and cerebellum. Sections from the lower brainstem showed no degeneration of the long tracts.

\section{Discussion}

The cases in the literature most closely resembling ours are compared with each other in the table. ${ }^{4-7}$

Several pathological entities need to be considered in the differential diagnosis of such cases, most importantly GerstmannStraussler-Scheinker syndrome, ${ }^{29}$ and Alzheimer's disease.

GSS is a familial, autosomal dominant disorder which begins around the age of 40, progressing over a period of about five years to death. Typical clinical features are prominent cerebellar ataxia, dementia, nystagmus, scanning speech, pseudobulbar signs, dysaesthesia of the legs, decreased lower extremity reflexes, and extensor plantar responses. ${ }^{289}$

Neuropathologically, in addition to cortical and cerebellar atrophy, GSS shows a unique spectrum of plaque morphology including multicentric and kuru-type plaques in the cerebellar cortex, and senile and primitive plaques in the cerebral cortex. ${ }^{8}$ Several cases have shown spongiform changes (see Note) in the cerebral cortex, with several of these GSS cases shown to transmit a spongiform encephalopathy. ${ }^{2}$ In addition some cases of GSS have also shown atrophy of multiple long tract pathways (referred to as systems atrophy). ${ }^{8}$

While our patient clinically resembled GSS in his dementia, decreased lower extremity reflexes, and extensor plantar responses, he clinically differed in the lack of family history, earlier onset, presence of rigidity and dystonia, lack of ataxia, and relative inconspicuousness of intention tremor. Neuropathologically, while the plaque morphology of GSS was present, there was also cortical neurofibrillary tangles, granulovacuolar degeneration in the hippocampus, and involvement of the substantia nigra and locus ceruleus, which have not been described in previous cases of GSS, except for those individual cases of uncertain assignment. ${ }^{47}$ Also the striatal plaques of our case have not been described in GSS.

In support of a categorisation as $\mathrm{Alz}$ heimer's disease, this case was not familial, and dementia was early and prominent. Up to $10 \%$ of $\mathrm{AD}$ patients have been reported to have rigidity and dyskinesia early in their course. ${ }^{10}$ However, the age of onset would of course be extremely atypical for AD. Nonetheless, the neuropathology of our case could have been considered typical $\mathrm{AD}$ in terms of cerebral atrophy, primitive plaques and senile plaques in the cortex, neurofibrillary tangles in the cortex, granulovacuolar degeneration in the hippocampus, and neuronal loss in the locus ceruleus. ${ }^{11}$ Its major neuropathological difference from $\mathrm{AD}$ was in the presence of multicentric plaques in the cerebral cortex and cerebellum, as are typical in GSS. Also, kuru-like plaques are common in GSS and only rarely described in $\mathrm{AD} .^{1}$

Given these overlaps, categorical distinctions among these conditions are perhaps not possible at this time (but $\mathrm{see}^{4}$ ). Immunohistochemical staining ${ }^{12}$ may ultimately prove valuable in differentiating these clinical entities.

Note Kuzuhara et al ${ }^{8}$ have drawn a distinction between "spongy" change and "spongiform" changes in the cortex, with the latter being the more prominent and widely distributed changes seen in classic CJD. So far, the only cases of GSS shown to be transmissible have had spongiform changes similar to those found in CJD. Not all cases of GSS with spongiform changes have been transmissible.

We thank Dr Edward Koo for neuropathological assessment, preparation of figures, and constructive comments on an preparation of

This study was supported in part by NINCDS TeacherInvestigator Award 1 K07 NS00721 to BG, and by Hopkins' Alzheimer's Disease Research Center grant NIA 1 P50 AG05146.

1 Pro JD, Smith CH, Sumi SM. Presenile Alzheimer disease: Amyloid plaques in the cerebellum. Neurology 1980;30: $820-5$.

2 Masters CL, Gajdusek DC, Gibbs CI. Creutzfeldt-Jakob disease virus isolations from the Gerstmann-Straussler disease virus isolations from the
syndrome. Brain 1981;104:559-88.

3 Tateishi J, Nagara H, Hikata K, Sato Y. Amyloid plaques in the brains of mice with Creutzfeldt-Jakob disease. $A n n$ Neurol 1984;15:278-80.

4 Azzarelli B, Muller J, Ghetti B, Dyken M, Conneally PM. Cerebellar plaques in familial Alzheimer's disease (Gerstmann-Straussler-Scheinker variant?). Acta Neuropatho (Berl) 1985;65:235-46.

5 Vinters HV, Hudson AJ, Kaufmann JCE. GerstmannStraussler-Scheinker disease: Autopsy study of a familia case. Ann Neurol 1986;20:540-3.

6 Kornfeld M. Gerstmann-Straussler syndrome with features of Alzheimer's disease: Two entities or one? J Neuropath Exp Neurol 1984;43:321.

7 de Courten-Myers G, Mandybur TI. Atypical GerstmannStraussler syndrome or familial spinocerebellar ataxia and Alzheimer's disease? Neurology 1987;37:269-75.

8 Kuzuhara S, Kanazawa I, Sasaki H, Nakanishi T, Shimamura K. Gerstmann-Straussler-Scheinker's disease. Ann Neurol 1983;14:216-25.

9 Gerstmann J, Straussler E, Scheinker I. Uber eine eigenartige hereditar-familiare erkrankung des zentralnervensystems: Zugleich ein beitrag zur frage des vorzeitigen lokalen alterns. Zeitschrift fur Neurologie 1936;154: 736-62.

10 Mayeux R, Stern Y, Spanton S. Heterogeneity in dementia of the Alzheimer type: Evidence of subgroups. Neurology 1985;35:453-61.

11 Bondareff W, Mountjoy CQ, Roth M. Loss of neurons of origin of the adrenergic projection to the cerebral cortex (nucleus locus ceruleus) in senile dementia. Neurology 1982;32:164-8.

12 Wolozin BL, Pruchnicki A, Dickson DW, Davies P. A neuronal antigen in the brains of Alzheimer patients. Science 1986;232:648-50. 